\title{
EAl Endorsed Transactions

\section{Secure Group based Routing and Flawless Trust Formulation in MANET using Unsupervised Machine Learning Approach for IoT Applications}

\author{
Maitreyi Ponguwala ${ }^{1, *}$ and DR.Sreenivasa $\operatorname{Rao}^{2}$ \\ ${ }^{1}$ Research Scholar of JNTUH, MGIT Hyderabad, India, Ponguwalamaitreyi@gmail.com \\ ${ }^{2}$ Professor in Dept. Of CSE, JNTUH Hyderabad, India, Srmeda@jntuh.ac.in
}

\begin{abstract}
INTRODUCTION: Mobile Adhoc Network (MANET) is integrated with Internet of Things (IoT) in many application cases due to its flexibility and scalability. The dynamic nature of MANET introduces some security threats in IoT environment. In those threats, Blackhole attack and Grayhole attack are severe routing attacks that disrupts routing algorithm to crack transmission in the entire network.

OBJECTIVES: Many security mechanisms are introduced in MANET based on trust computation schemes. However, computation of inaccurate trust value degrades the performance of mitigation schemes. Thus the major objective of this work is to design a novel security mechanism to protect the MANET-IoT from different adversaries.

METHODS: in this paper we propose a novel group based routing algorithm with recommendation filtering supported by security monitors (SMs). Unsupervised machine learning algorithm is adapted for the purpose of recommendation filtering in the network. Initially the entire network is grouped by Secure Certificate based Group Formation (SCGF) algorithm. In each group, Recommendation Filtering by K-means algorithm (RF-K means) algorithm is employed to perform trust computation. For secure and optimal route selection, hybrid optimization algorithm that combines Genetic Algorithm and Fire Fly Algorithm (GA-FFA) is proposed. Data transmission is protected by novel Hash Message Authentication Code with Advanced Encryption Standard (HMAC-AES) algorithm in which hash function is integrated with cryptography function.

RESULTS: The proposed work is validated in network simulator-3 environment and the obtained results show better performance in terms of packet delivery ratio $(96.3 \%)$, throughput $(135 \mathrm{kbps})$, delay $(3.26 \mathrm{~ms})$, detection rate $(99 \%)$, and energy consumption $(8.5 \%)$.

CONCLUSION: The MANET-IoT network is secured by group formation and trust filtering approaches. Further, involvement of cryptography function ensures data security whereas hash function ensures data integrity..
\end{abstract}

Keywords: MANET, Recommendation Filtering, Blackhole, Grayhole, IoT applications.

Received on 20 March 2019, accepted on 03 August 2019, published on 09 October 2019

Copyright (C) 2019 Maitreyi Ponguwala et al., licensed to EAI. This is an open access article distributed under the terms of the Creative Commons Attribution licence (http://creativecommons.org/licenses/by/3.0/), which permits unlimited use, distribution and reproduction in any medium so long as the original work is properly cited.

doi: 10.4108/eai.13-7-2018.160834

"Corresponding author. Email: maithriponguwala@gmail.com

\section{Introduction}

MANET is an infrastructure-less, dynamic, and distributed network that has self-configurable ability [1]-[3]. MANET comprises group of mobile nodes that establish communication in an opportunistic manner. It follows an infrastructure-less architecture (i.e.) MANET doesn't lies on centralized base station or sink node. The specific characteristics of MANET are: multi-hop communication, decentralized infrastructure, dynamic topology, scalability, and short range connectivity. Rapid growth in wireless 
technologies increases requirement of IoT nowadays [4], [5]. IoT can be described as a network that comprises millions of heterogeneous devices and enables communication among these smart 'things'. Empowering opportunistic communication among IoT devices becomes significant since IoT involves with dynamic devices. In order to enable opportunistic communication among IoT devices MANET is integrated with IoT [6], [7]. Immersion of MANET in IoT brings improved connectivity through multi-hop communication and also supports high scalability. With the integration of MANET, IoT applications can be extended in military scenarios, smart city applications, waste management, and disaster management and so on. However, absence of the infrastructure and centralized control increases the presence of security threats in MANET-IoT environment [8], [31]. MANET is vulnerable to security attacks such as jamming attack, Grayhole attack, rushing attack, Blackhole attack, packet dropping attack, and Sybil attack. These attacks degrade the performance of entire network.

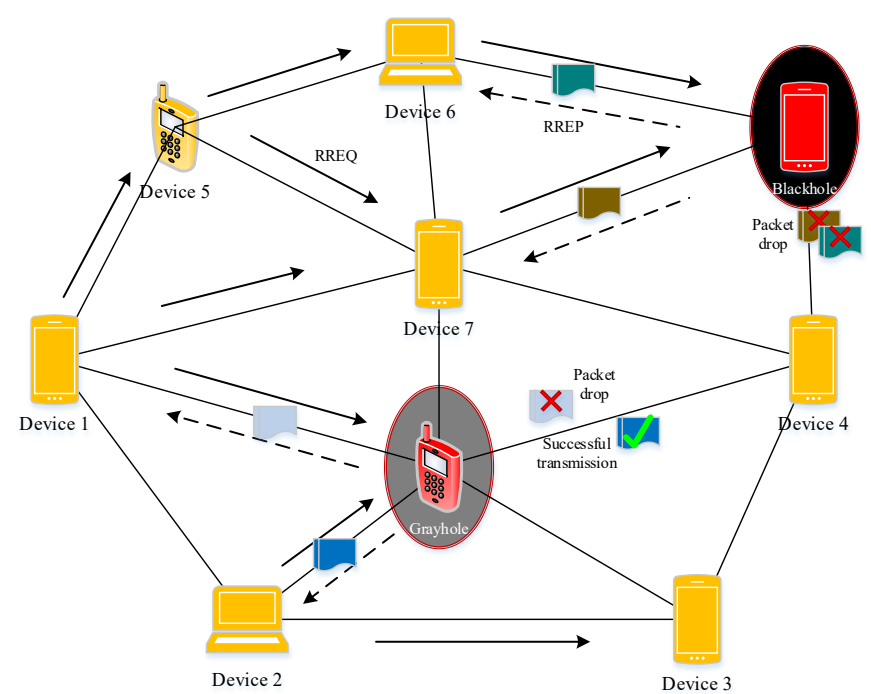

Figure 1. Blackhole and Grayhole attack in MANETIoT

Blackhole attack and Grayhole attack are moreover similar routing attacks in MANET-IoT [9], [10]. Both attackers involved in malicious activities such as packet dropping, packet altering, packet redirecting, route alteration, and so on. In figure.1, the behavior of Blackhole attack and Grayhole attack is illustrated. Here the Blackhole attacker receives the data by sending false routing information to source node. It prevents data from reaching destination through dropping received data. This will introduce huge packet loss in the network. Similarly, Grayhole attack is a variant of Blackhole attack that drops only selective packets. In other words, the Grayhole attacker drops selective packets and forwards remaining packets to the destination. In the figure, the Grayhole attacker drops data from Device 1 and forwards the data from Device 2. Majority of
Blackhole and Grayhole attack mitigation schemes utilize trust based methods for attack detection and prevention [32]. In order to mitigate Blackhole attack in MANET a data control packet and extended data routing information table (EDRI) are used in adhoc on-demand distance vector (AODV) protocol [11]. Herein routing path verification process is enabled for attack detection. Mitigation of Blackhole attack in IoT environment is carried out by securing routing protocol over low power lossy network (RPL) protocol [12]. In RPL, destination oriented directed acyclic graph (DODAG) construction is enabled with trust value computation. A flooding factor based framework for trust management (F3TM) is proposed to mitigate Grayhole attack in MANET through trust value computation [13]. For validating network nodes, a grey wolf and swarm optimizer algorithm is introduced. Cryptography schemes are also concentrated to provide security in MANET and IoT [33]. A selective significant data encryption (SSDE) algorithm utilizes Blowfish algorithm [14]. However, the cryptography technique must minimize the overhead and time consumption (i.e.) lightweight cryptography techniques are most suitable for MANET.

\subsection{Major Contributions}

Many research works are held on IoT and MANET in the perspective of security. But attack detection with data security and integrity is still challenging issue with the increase in number of IoT devices as well as number of adversaries in MANET-IoT environment. To cope with these issues, this paper presents following contributions,

- A novel IoT architecture is designed with the integration of MANET for establishing group based communication. A new entity namely Security Monitor (SM) is deployed in the network to strengthen the security level. Security provisioning is achieved by secure group formation, flawless trust value computation, trusted routing and partial encryption processes.

- Group formation is initialized with group head (GH) selection by SM. SCGF algorithm is proposed to form secure groups in the network. SCGF algorithm not only ensures network security but also supports dynamic mobile network management. Secure grouping process isolates unauthorized nodes in network.

- $\mathrm{SM}$ is responsible for flawless trust computation by using RF-K means algorithm. Unsupervised Kmeans algorithm is adapted for trust computation through recommendation filtering mechanism. By formulating optimal trust computation value, the Grayhole attack detection is performed.

- Trusted route selection is accomplished by effective hybrid optimization algorithm named as GA-FFA algorithm. Trusted routing process supports Blackhole attack detection through routing reply analysis. Route selection by GA-FFA algorithm is not only trustworthy but also efficient 
since fitness computation involves with trust value, residual energy, link stability, and hopcount.

- Data security and integrity is achieved jointly by HMAC-AES algorithm. HMAC-AES algorithm performs based on partial encryption and partial hashing process in order to minimize encryption time and overhead without loss in security and integrity.

\subsection{Paper organization}

The rest of this paper is organized as follows: Section II surveys significant related works held on IoT and MANET for security provision. In section III, the problems existed in previous research works are highlighted. Section IV, details overall process of proposed security mechanism with novel algorithms. Section V, evaluates the proposed work in terms of performance metrics and compares proposed work with previous works. In section VI, we conclude our contributions and highlight the future directions of this work.

\section{Related Works}

In past few years, enormous numbers of research works are held on MANET and IoT for security provisioning. In this section, we provide detailed literature review on significant previous research work in order to exploit the vital challenging issues in this research area.

\subsection{Survey on attack detection methods}

In adhoc networks, Blackhole attack detection was performed by fuzzy based trust value computation [15]. Trust value of each node was computed in fuzzy approach based on following metrics: packet delivery ratio, percentage of energy exhaustion, percentage of buffer utilization, and number of connection requests given by a node. If computed trust value was greater than threshold value then that node is considered as normal otherwise that node is considered as Blackhole attacker. However, here node behavior analysis is performed by fuzzy approach (i.e.) threshold computation is an additional process which increases time consumption. Blackhole attack detection was performed based on dynamic threshold cumulative sum (CUSUM) test [16]. Here CUSUM test was utilized in order to detect the changes in normal behavior of AODV's sequence number. Based on AODV's sequence number (SQN), a new parameter named as Blackhole intensity was formulated for attack detection. Perhaps this method performs attack detection; this method is not able to secure transmitted data.

Blackhole attack, Grayhole attack and flooding attack were mitigated jointly by multi-level trust computation scheme [17]. Here Elliptic Curve Cryptography was used and the security was categorized into three levels. The first level computes direct trust value and indirect trust value, then in second level objective and subjective trust was computed and lastly in third level static and dynamic trust was estimated. Summing up of all the three levels of trust, the total trust value was obtained. Although ECC algorithm provides data security, it is not able to ensure data integrity which leads to data alteration. For Grayhole attack detection, denial contradictions with fictitious node mechanism (DCFM) was proposed [18]. The original DCFM algorithm was developed to mitigate node isolation attack in Optimized Link State Routing (OLSR) based routing algorithm. Then the algorithm was improved to extend the attack detection to Grayhole attack. This method was able to handle passive silent attacker, randomly located attacker, initially 1-hop neighbor attacker, shadow attacker, and man in the middle attacker. This method increases complexity while fails to improved detection accuracy. A trusted AODV (TOAV) protocol was presented to mitigate routing attacks in MANET [34]. Herein trust value was represented by opinion. An acknowledgement based method namely $2 \mathrm{ACK}$ method was proposed to detect misbehavior in MANET environment [35]. This method was implemented on the top of dynamic source routing (DSR) protocol. In MANET, trust value of node was defined based on mobility, pause time, and remaining battery power [36]. However, consideration of inappropriate trust metrics is inefficient for providing security in MANET. Thus in majority of works, data security is not concentrated which increases vulnerability of data.

\subsection{Survey on security mechanisms}

AODV routing protocol was extended with level of trust (LOT) value [19]. Here LOT computation was performed by fuzzy approach. In fuzzy logic, trust value of node and throughput were taken as input and required encryption level was obtained as output. If an intermediate node has lower trust value and lower throughput then it requires high encryption. Then source encrypt the data and send the encrypted data to relay node. In this manner data security was achieved in this work. The transmitted data is subjected to alterations since data integrity is not ensured. In addition, packet dropping and packet redirecting attack are also held on the network. For securing group communication, three clustering algorithms were presented in MANET [20] as follows: (i) simple clustering algorithm, (ii) weighted clustering algorithm, and (iii) fuzzy clustering algorithm. In simple clustering algorithm, cluster formation is performed based on single metric known as connectivity. In weighted clustering algorithm, connectivity, energy, mobility, and distance were considered for cluster formation. In fuzzy based clustering, trust value of a node was computed in terms of number of successful transactions. Then in each cluster, group key was generated and distributed among CMs. However, $\mathrm{CH}$ selection by considering single metric (trust value) demands frequent $\mathrm{CH}$ selection in dynamic environment. Trust value computation based on number of successful transmissions is also not efficient. 
To improve security against multiple threats, support vector machine (SVM) based intrusion detection system was proposed for ad-hoc networks [21]. In SVM classifier, average packet drop, average packet reception, and average packet interval were considered for attack detection. However, SVM is a supervised machine learning algorithm which requires huge data size and large time for training. In IoT environment, privacy and security was ensured by incorporating message authentication code (MAC) [22]. For that, a privacy-preserving communication protocol was designed with MAC. However, computing hash function for whole data increases computational complexity as well as memory consumption. In addition, this method is only able to provide data integrity and data security is not provided. In IoT environment, security was ensured by robust ECC based authentication protocol [23]. In this approach, each sensor was registered and authenticated through ECC protocol. Following security services were provided by this method: anonymity, untracebility, forward secrecy, and backward secrecy. Perhaps, this protocol provides authenticity; this method is not able to ensure data security and integrity. In IoT network, communication between nodes and server was secured by heterogeneous ring signcryption scheme [24]. The major objective of this scheme was to defend against ciphertext attacks in the network. This scheme increases time consumption with increase in number of nodes.

Thus all previous research works consider trust value for attack detection in ad-hoc network. However, the trust computation is also vulnerable since malicious node can modify this trust value. Similarly, none of these works is able to achieve data security and data integrity jointly without increase in computational complexity.

\section{Problem Definition}

In this section, we exploit major problems identified in MANET and IoT for security provisioning. Trusted security AODV (TS-AODV) protocol was designed with IDS to mitigate flooding attack, Blackhole attack, and Grayhole attack [25]. In IDS, a source-trust-rate was provided based on number of route requests generated. Here trust value for source node was computed as follows,

$$
\text { Source }- \text { Trust }=(\text { RREQ Count })^{-1}
$$

In eqn.(1), trust value for source node was computed based on number of RREQ packets forwarded by that node. However, trust value computation is ineffective since in AODV, each intermediate node broadcast RREQ packet for route selection. Furthermore, route selection based on trust value alone increases number of retransmissions due to frequent link breakages. Fuzzy logic based trust management mechanism were proposed in MANET [26], [27]. In fuzzy based OLSR routing, packet delivery ratio (PDR), and delay were considered for trust computation. However, both PDR and delay metrics are not only affected by malicious activities but also affected by other factors. Thus trust computation based on PDR and delay is not effectual. Fuzzy based secure architecture (FBSA) was comprised with training and testing phases. Based on fuzzy rules, the network nodes were categorized into malicious, normal, and best node. Here malicious activity is identified by fuzzy rules in which involvement of training phase increases time consumption. In addition, this method is able to identify malicious activities only; not able to detect malicious activities exactly. For mitigating Blackhole attack, a secure-AODV (SAODV) protocol was designed [28]. In SAODV, the first RREP packet received by source node was dropped and the node which sends RREP packet was considered as Blackhole attacker. Nonetheless, it is not ensured that first RREP packet is always malicious. Thus dropping first RREP request is not an intelligent wayto mitigate malicious attack. In addition, SAODV detects only affected path instead of detecting Blackhole attacker.

The problems highlighted in this section are considered in our work and resolved by our novel approaches. The major problems identified in this section are: (i) trust management is not accurate, (ii) route selection is not effectual, and (iii) attack detection is not efficient. Furthermore, data security and integrity is not ensured in all above works.

\section{Proposed Secure MANET-IoT}

\subsection{Network Overview}

To improve security and integrity, we design a novel group based routing scheme and flawless trust formulation for MANET-IoT environment. The overall proposed network environment is illustrated in figure.2. Our network environment comprises following entities: $n$ number of MANET-IoT devices/nodes as $N=N_{1}, N_{2}, \ldots, N_{n}$, certificate authority (CA), $k$ number of security monitors $S M=$ $\left\{S M_{1}, S M_{2}, \ldots, S M_{k}\right\}$, and malicious devices. Security monitors are responsible for malicious node detection, recommendation filtering and secure group formation. Initially, the devices are grouped by security monitors after certificate verification. For flawless trust value formulation, security monitors utilize RF-K means algorithm. Further, route selection is involved with hybrid optimization algorithm which is effectively designed by combining GA and FFA algorithm with new fitness formulation. Data security and integrity are assured by HMAC-AES algorithm during data transmission. Each significant process is explained in following subsections.

\subsection{Secure Group Formation}

Integration of MANET and IoT to design MANET-IoT supports many applications and has many advantages. However, mobility management in MANET-IoT becomes challenging issue since the network topology is dynamically changed due to dynamic mobility of devices. In order to manage this kind of dynamic environment, grouping is an effectual solution. Thus we perform grouping of IoT devices by using SCGF algorithm. 


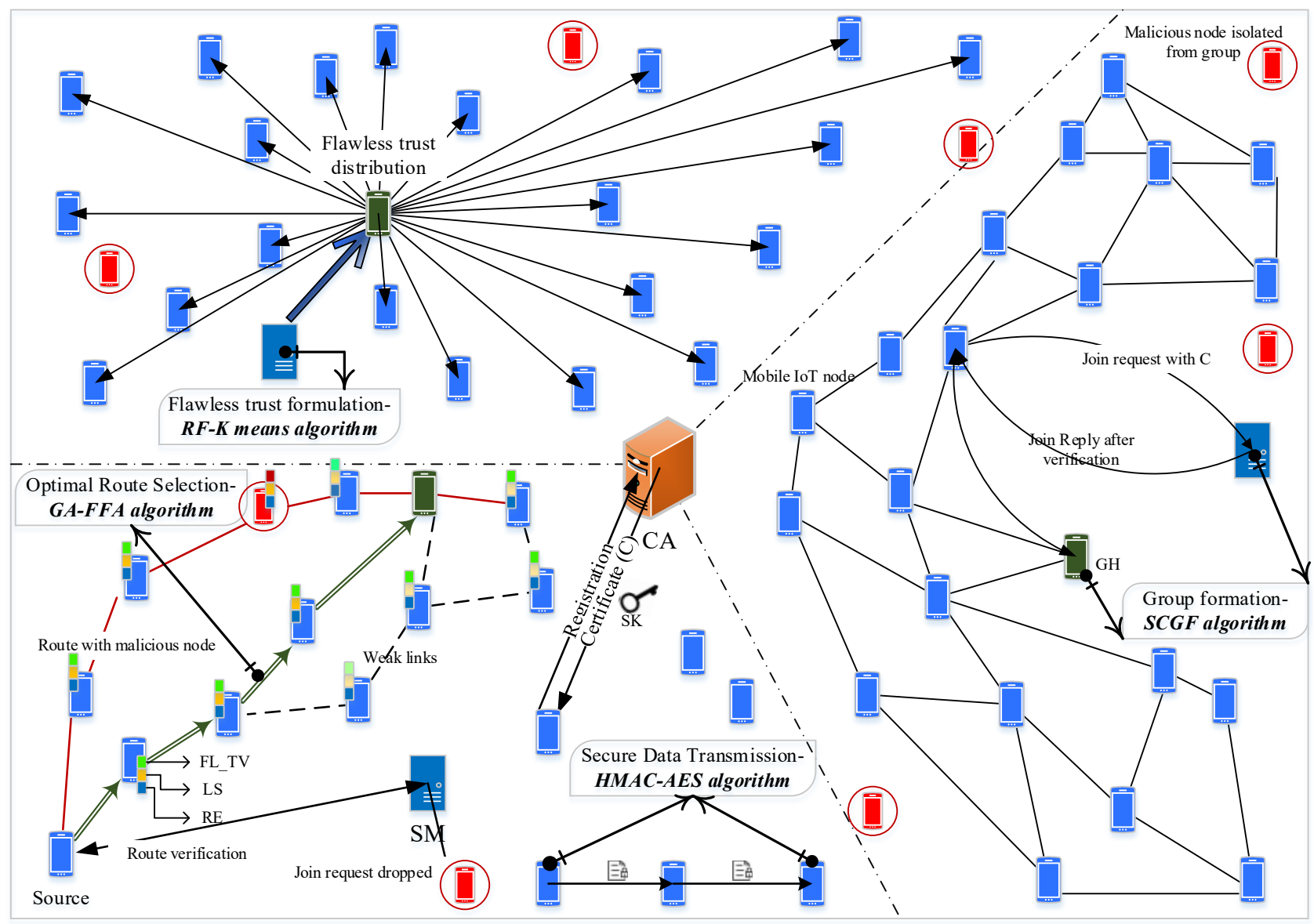

Figure 2. Proposed Secure MANET-IoT environment

Involvement of SCGF algorithm improves network management as well as security of the network through certificate verification. Initially, the network is divided into regions based on security monitors. The nodes within the range of each SM belong to corresponding group that is formed by that SM. SCGF algorithm is initiated with optimal GH selection. For optimal GH selection, weighting factor $(W F)$ is formulated as follows,

$$
W F\left(N_{i}\right)=E_{N_{i}}-\left(M_{N_{i}}+A D_{N_{i}}\right)
$$

The weighting factor of ' $i$ th, node $N_{i}$ is computed based on energy level $\left(E_{N_{i}}\right)$, mobility $\left(M_{N_{i}}\right)$, and average distance with neighbor nodes $\left(A D_{N_{i}}\right)$. A node with highest $W F$ in particular region is selected as GH by $S M$. Then the selected $\mathrm{GH}$ is verified through certificate verification. For this purpose, all IoT nodes must be registered with $\mathrm{CA}$ in order to obtain certificate $(C)$ and secret key $(S K)$ to form secure group. Each node submits its unique ID (ID) and password $(P W)$ to CA during registration. Then the CA provides a digital certificate to that corresponding node. The digital certificate consists of ID of the node, signature of CA $(\operatorname{Sign}(C A))$, and secret key of node, and unique certificate ID $(I D(C))$. After the completion of registration, CA distributes the certificates of registered nodes to $S M S$ for further verification. The selected GH submits its $\{I D \| C\}$ to $S M$ in order to receive group secret $(G S)$. When $\mathrm{GH}$ submits the $\{I D \| C\}, S M$ verifies whether the node is legitimate or not. For verification, $S M$ checks the $\operatorname{Sign}(C A)$ in $C$ and encrypt the $G S$ by using secret key of GH. If the node is legitimate $S M$ sneds $G S$ to that node in encrypted form $(E n\{G S\})$ and broadcast the $G H$ selection message to all other nodes in that region. Thus the node must decrypt the $G S$ in order to join with group. Upon receiving, $G H$ selection message, all other nodes sends JoinReq to GH. However, for group formation the node must have $G S$ obtained from $S M$ (i.e.) the node must be verified by $S M$. If $G S$ submitted by node is matched with $G S$ provided by $S M$, then $G H$ sends JoinRep to that node. Otherwise, $G H$ deny the access of that node from group formation. Thus in SCGF algorithm, the legitimate nodes in the region form secure group to establish communication among nodes. Here malicious nodes are isolated form the group through verification.

In figure.3, the process of SCGF algorithm is illustrated. Thus involvement of SCGF algorithm isolates malicious and non-legitimate nodes in each region through secure group formation. Secure group formation 
supports dynamic network management and also supports flawless trust formulation through recommendation filtering.

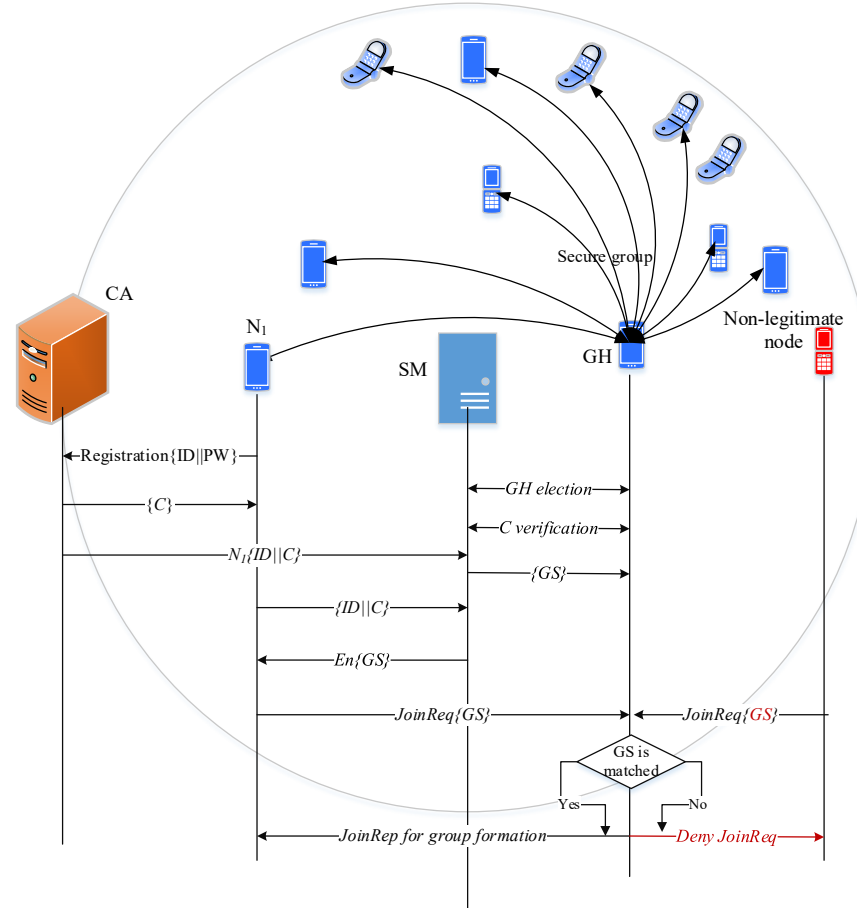

Figure 3. SCGF algorithm based group formation

\subsection{Flawless Trust Formulation}

In MANET-IoT network, majority of attackers are identified by analyzing trust value. Thus trust value computation plays vital role in security provisioning in MANET-IoT. However, trust value is also subjected to security threats such as trust value distortion attacks. In this type of security threats, the malicious nodes alter the trust value of other nodes during indirect trust computation which introduces unnecessary conflicts in the network. In addition, inaccurate trust computation leads to ineffectual attack detection. In our work, we adapt RF-K means algorithm for flawless trust formulation as well as for Grayhole attack detection. RF$\mathrm{K}$ means algorithm is performed by $S M$ for trust computation. For each node, $S M$ computes flawless trust value $\left(F L_{-} T V\right)$ based on trust value provided by other nodes. Thus for each node $F L_{-} T V, S M$ performs RF-K means algorithm. The process of RF-K means algorithm in flawless trust formulation and Grayhole attack detection is described in following steps,

Step 1: In this step, $S M$ collects $T V$ from all nodes in the region through GH. In our work, each node computes $T V$ for its neighboring nodes and transmits it to corresponding $\mathrm{GH}$ periodically. Then $\mathrm{GH}$ aggregates the $T V$ and sends $T V$ to $S M$. The $T V$ that is transmitted to $S M$ is in the form of $\left[N_{1} \rightarrow\{T V s\}, N_{2} \rightarrow\{T V s\}, \ldots, N_{j} \rightarrow\right.$ $\{T V s\}]$ (i.e.) the node ID $\left(N_{1}\right)$ and its corresponding trust values are provided by other nodes (TVS). Each node computes $T V$ of neighboring nodes as follows,

$$
T V=\frac{T R}{B S+D R}
$$

For a node $T V$ is computed in terms of transmission rate $(T R)$, buffer size $(B S)$, and drop rate $(D R)$. Transmission rate of a node $(T R)$ is computed as follows,

$$
T R=\frac{\text { Number of packets forwarded }}{\text { Total NUmber of packets sent }}
$$

Similarly $B S$ and $D R$ can be calculated as follows,

$$
\begin{gathered}
B S=\frac{\text { Buffer Utilized }}{\text { Buffer Size }} \\
D R=\frac{\text { Number of packets dropped }}{\text { Total number of packets sent }}
\end{gathered}
$$

Step 2: In this step, $S M$ performs RF-K means algorithm for each node based on TVS provided by other neighboring nodes. For this purpose, $S M$ initializes number of clusters $K$ value. Thus for each node, $K$ number of clusters are formed for TVs.

Step 3: Randomly initialize number of centroid as follows,

$$
\text { Centroids }=\left\{T V_{C 1}, T V_{C 2}, \ldots, T V_{C K}\right\}
$$

Here the centroids initialized are trust values of a node. Let $N_{1}$ has $T V S$ as $T V=\left\{T V_{1}, T V_{2}, \ldots, T V_{L}\right\}$. Thus from this set of $T V s, K$ number of centroids are initialized in random manner.

Step 4: Assign TVs to nearest centroid based on trust distance (Tdis) value. Here the Tdis is computed as the difference between $T V$ and centroid. For ' $k$ th, $T V$, the difference with ' $j^{\text {th }}$ ' centroid is computed as,

$$
\begin{gathered}
\text { Tdis }=\left|T V_{C j}-T V_{k}\right| \\
\text { If } \operatorname{Tdis}(j, k)=\text { Small, } \operatorname{assign} T V_{K} \rightarrow T V_{C j}
\end{gathered}
$$

Eqn.(9) represents that the $T V_{k}$ is assigned to centroid which minimizes Tdis. In this manner, all TVs are assigned to closest centroid.

Step 5: In this step, all centroids are updated (i.e.) recomputed. Then all above steps are performed with new centroid until optimal ' $K$ ' clusters are formed. In this step ' $K$ ' number of recommendation clusters $(R C l s)$ are formed as,

$$
R C l s=\left\{R C l s_{1}, R C l s_{2}, \ldots, R C l s_{K}\right\}
$$

Step 6: In this step, weighted inter-cluster difference is computed in order to exploit Grayhole attack detection. Typically Grayhole attacker is a selective forwarding attacker which forwards data from selective sources and drops data from other nodes. In this case, trust value 
which is computed by particular node is too large while by other nodes are low for an attacker. Thus there is high possibility to the attacker to be considered as honest node. In order to avoid this conflict, RF-K means algorithm performs inter-cluster distance computation. Before that, each $T V$ in each cluster is weighted based on following function,

$$
\begin{gathered}
W\left(T V_{j}\right)=\frac{\sum_{i=1}^{X} \Delta\left(T V_{j}, T V_{i}\right)}{X} \\
\text { Weighted } T V_{j}=T V_{j} \times W\left(T V_{j}\right)
\end{gathered}
$$

Here $X$ represents number of $T V S$ in the cluster and $\Delta\left(T V_{j}, T V_{i}\right)$ represents difference with ' $i$ ' $T V$. In this manner, each trust value is computed as weighted $T V$ in this step. After completion of weighted TV computation, average weighted $T V$ is computed for each cluster as follows,

$$
\text { AvgTV }=\frac{\sum_{j=1}^{X} \text { Weighted } T V_{j}}{X}
$$

This average TV is computed for each cluster in RCls. Then inter-cluster cluster distance is computed as follows,

$$
I C d=\left|R C l_{1}-R C l_{2}\right|
$$

Eqn.(14), represents the inter-cluster distance (ICd) between two clusters $R C l_{1}$ and $R C l_{2}$. If this distance is small, then the node is not a Grayhole attacker. Otherwise the node is considered as Grayhole attacker since Grayhole attacker obtains high $T V$ from particular nodes and obtains low $T V$ from other nodes.

Step 7: In this step, flawless $T V$ computation is performed based on majority rule. For each $F L_{-} T V$ is computed as AvgTV of cluster with large number of TVS (i.e.) the $T V$ which is provided by majority of nodes is assigned as $F L_{-} T V$ as follows,

$$
F L_{-} T V(j)=\operatorname{AvgTV}(j), \text { if } X=\text { larger }
$$

Thus in RF-K means algorithm, Grayhole attacker is detected effectually and also $F L_{-} T V$ computation is accomplished. The computed $F L_{-} T V$ for each node is distributed to all other nodes in the group through $\mathrm{GH}$. Then this $T V$ is further used for optimal route selection.

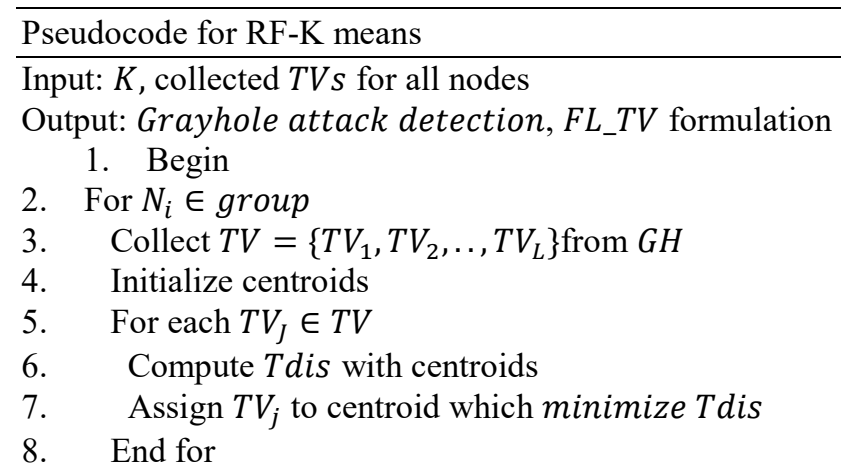

9. Update centroids

10. Form $R C l s=\left\{\right.$ RCls $\left._{1}, \mathrm{RCls}_{2}, \ldots, \mathrm{RCls}_{K}\right\}$

11. For each $T V \in R C l$

12. Compute $W(T V)$ using (11)

13. Find Weighted TV using (12)

14. End for

15. For all $\mathrm{RCls}$

16. Compute AvgTV

17. Find $I C d$

18. If $I C d==$ Low

19. $N_{i}$ is a Grayhole attacker

20. Else

21. $N_{i}$ is honest

22. End if

23. Compute FL_TV using (15)

24. End for

25. End for

26. End

The above pseudocode explains the procedure of proposed RF-K means algorithm. This algorithm can be explained through an example. Consider a node $N_{i}$ has three neighbour nodes as $N_{i+1}, N_{i-1}$, and $N_{i+2}$. Each node provides TVS for $N_{i}$ as follows, $N_{i+1} \rightarrow 10, N_{i-1} \rightarrow$ $11, N_{i+2} \rightarrow 2$. In this case, two RCls are formed as $R C l_{1}=\{10,11\}$ and $R c l_{2}=\{2\}$. The Weighted $T V$ in each cluster is computed as $R C l_{1}=\{5.25\}$, and $R C l=$ $\{2\}$. Thus $I C d=3.25$. Here inter-cluster distance is high, thus the node is considered to be an attacker (i.e.) $N_{i}$ forwards legitimately from $N_{i+2}$ and drops packets from other nodes. In this manner, Grayhole attack detection is carried out by RF-K means algorithm.

\subsection{Hybrid optimization algorithm for secure routing}

In this section, we propose a hybrid optimization algorithm for secure route selection for data transmission. Involvement of adversaries in the network increases the packet loss in the network. To secure data transmission, it is necessary to select trusted path between source and destination. However, MANET-IoT is highly dynamic which also requires optimal route for data transmission. Thus we propose GA-FFA algorithm for optimal route selection between source and destination. GA is a wellknown algorithm that has been employed for optimal route selection in MANET [29]. Although GA provides optimum route for data transmission, it consumes large time for convergence. On the other hand, FFA algorithm shows efficiency in route optimization for MANT [30]. In order to mitigate the issue of GA, a new FFA algorithm is integrated with GA for route selection. In GA-FFA algorithm, multiple available paths between source (Src) and destination (Des) are generated by GA and an optimal route is selected by FFA algorithm. In GA algorithm, the available paths are generated by applying crossover and mutation operators. Then these available paths are 
initialized as fireflies in FFA algorithm. In all available paths, route request (RREQ) is sent by $\operatorname{Src}$ and route reply $(R R E P)$ is sent by intermediate nodes. In order to detect Blackhole attacker, the sequence number (SeqNum) of all RREP packets is analyzed. If SeqNum difference is large, then the node which sent RREP packet $\mathrm{s}$ considered as malicious node. Then this route is eliminated from available paths. After elimination of attacker path, finally the following multiple paths are initialized in FFA algorithm,

$$
A P=\left\{R_{1}, R_{2}, \ldots, R_{Y}\right\}
$$

In GA, $Y$ number of routes are generated between $S r c$ and Des. Then all available paths $(A P)$ are initialized in FFA algorithm.

In FFA algorithm following rules are deployed for optimal route selection,

- All fireflies are unisex (i.e.) attracts each other regardless of their gender

- Attractiveness is directly proportional to brightness and distance is inversely proportional to both attractiveness and brightness

- Objective function is used to determine the brightness level.

Based on above rules, the process of FFA for optimal route selection is performed as follows,

1. Formulation of objective function $(O F)$ as follows,

$$
O F=\operatorname{Max}\left(\frac{F L_{-} T V, R E, L S}{H C}\right)
$$

The objective function of our proposed GA-FFA algorithm is to select optimal route that maximizes $F L_{-} T V, R E$, link stability $(L S)$, and minimizes hopcount $(H C)$.

2. Initialize all available routes $A P=\left\{R_{1}, R_{2}, \ldots, R_{Y}\right\}$ fireflies in the population.

3. Computation of light intensity $(I(R))$ is performed based on $O F$ as follows,

$$
I \propto O F(R)
$$

4. Attractiveness between two fireflies is formulated as follows,

$$
\beta=\beta_{0} e^{-\gamma r^{2}}
$$

The attractiveness is determined based on light absorption coefficient $(\gamma)$, and attractiveness at $r=0$ $\left(\beta_{0}\right)$, and the distance between those fireflies $(r)$.

5. Movement of firefly towards more attractive firefly (i.e.) optimal solution is determined as,

$$
x_{i}=x_{i}+\beta_{0} e^{-\gamma r}\left(x_{j}-x_{i}\right)+\alpha \varepsilon
$$

6. Update light intensity based on movement and rank the fireflies accordance with light intensity.

7. Select optimal route $(O R)$ over iteration

8. End the process

By executing all above steps, the GA-FFA algorithm selects optimal route $(O R)$ between $S r c$ and Des. In GAFFA algorithm $O F$ is formulated based on significant routing metrics which improves security as well as efficiency of data transmission. Selection of optimal route based on FL_TV minimizes the possibility of adversaries in the particular route. Thus proposed GA-FFA algorithm ensures high level security as well as data transmission efficiency in MANET-IoT network.

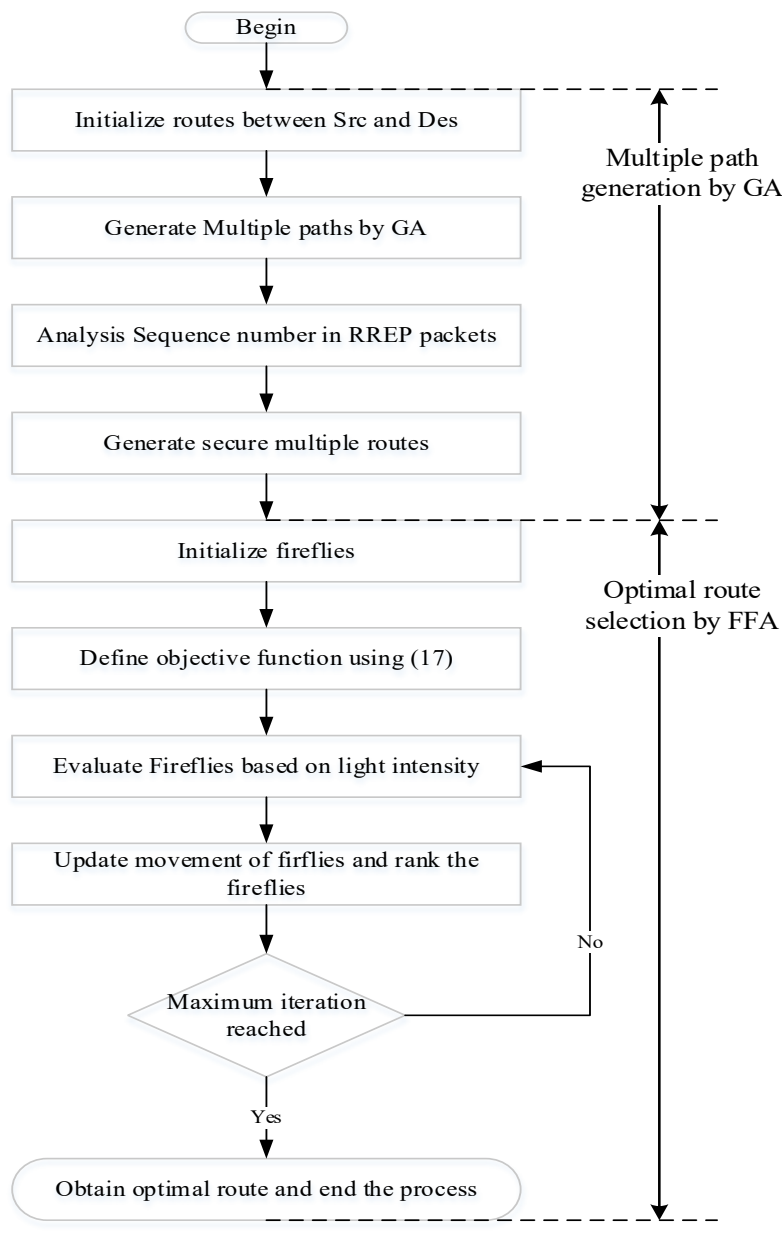

Figure 4. Process of GA-FFA algorithm

In figure.4, the entire process involved in GA-FFA algorithm is illustrated. Here GA algorithm generates multiple available paths and the affected paths are eliminated by sequence number analysis. Then the secure routes which are free from Blackhole attack are initialized in FFA algorithm to identify optimal route between $\operatorname{SrC}$ and Des. 


\subsection{Data security and integrity provisioning}

Perhaps, our proposed network is secured through secure clustering and routing; data security and integrity are still required for transmitted data to improve security level. To achieve data security and integrity jointly, we proposed a novel method that integrates hash function and cryptography function without increase in overhead and time consumption. For ensuring data security and data integrity, HMAC-AES algorithm is proposed. In HMACAES algorithm, the data $(D)$ to be transmitted is divided into two equal blocks as $\left\{D_{1}, D_{2}\right\}$. Then the first block of the data $\left(D_{1}\right)$ is encrypted by AES algorithm and the second data block $\left(D_{2}\right)$ is involved with has generation by HMAC algorithm. AES algorithm involves four major transformations such as SubBytes, ShiftRows, MixColumns, and AddRoundKey. Here encryption of data is performed through key expansion processes and each round key is stored in 4-byte words denoted as $w[z]$ In AES algorithm data encryption is performed as follows,

\section{Pseudocode for AES encryption}

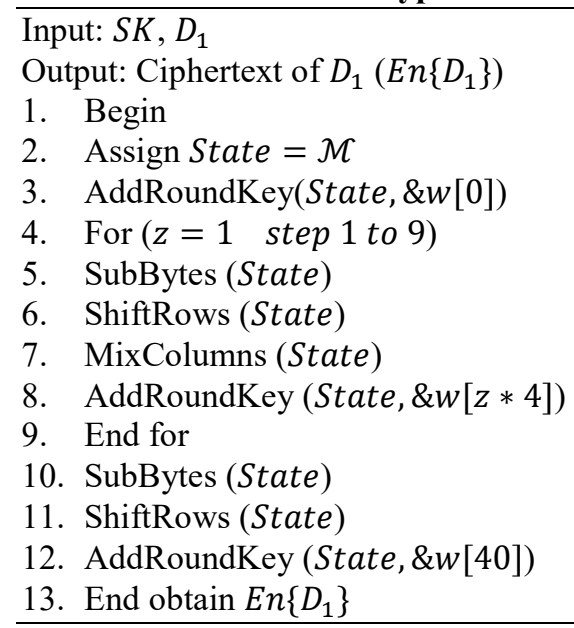

The above pseudocode explains the process of AES algorithm in data encryption. Here the first part of the data is encrypted and corresponding ciphertext $\left(\operatorname{En}\left\{D_{1}\right\}\right)$ is obtained. Forming data block, message authentication code (MAC) is generated to ensure data integrity using HMAC algorithm. HMAC based MAC generation is performed as follows,

$H M A C\left(S K, D_{2}\right\}=H\left(\left(K^{\prime} \oplus\right.\right.$ opad $) \| H\left(\left(K^{\prime} \oplus\right.\right.$ ipad) $\| m)$ )

The overall process of HMAC based MAC generation is performed illustrated in following pseudocode. Thus in HMAC-AES algorithm the first data block is encrypted by AES algorithm and foe second data block MAC is generated by HMAC algorithm. Then the data is transmitted to destination in the form of $\left(\left\{E n\left\{D_{2}\right\}, M A C\left\{D_{2}\right\}\right)\right.$ (i.e.) ciphertext of $D_{1}$, MAC of $D_{2}$, and plaintext of $D_{2}$. In destination, the ciphertext is decrypted using AES algorithm and the MAC in received data is verified by generating MAC for $D_{2}$.

\section{Pseudocode for HMAC based MAC generation}

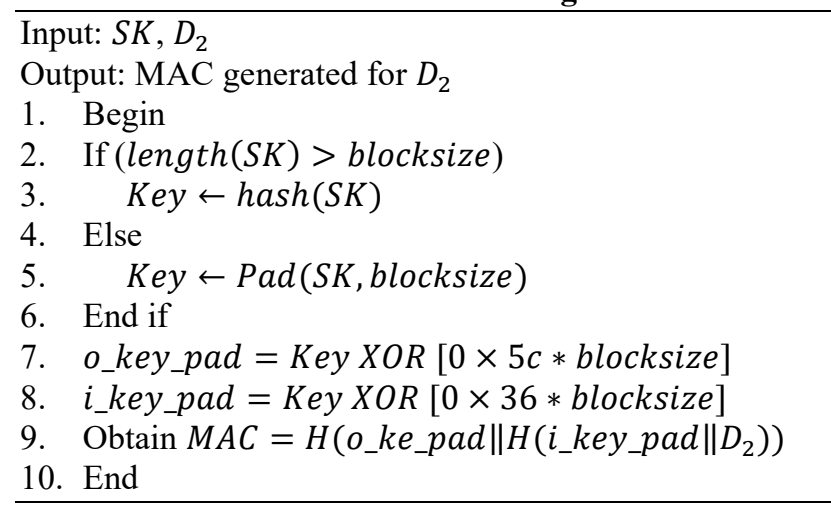

Thus AES algorithm provides data security whereas HMAC algorithm ensures data integrity. If any attacker obtains the data, the attacker is not able to obtain entire data without secret key. Similarly, the alterations made by attacker can be determined by HMAC algorithm. Thus our proposed work ensures both data security and data integrity for transmitted data.

\section{Experimental Results and Simulation}

In this section, we evaluate our proposed work with previous research works through necessary simulations. In this section, simulation environment of proposed secure MANET-IoT is briefly described and the comparative analysis is performed to measure the efficiency of our proposed work.

\subsection{Simulation environment of MANET-IoT}

Our proposed secure MANET-IoT that integrates MANET in IoT environment is simulated in network simulator-3.26 (ns-3.26) which is a discrete event simulator that supports various communication standards, and different networks. Our network comprises MANETIoT devices, CA, SMs, and malicious nodes.

Table 1. Simulation parameters

\begin{tabular}{ll}
\hline Parameter & Value \\
\hline Simulation area & $1000 \times 1000 \mathrm{~m}$ \\
Number of loT devices & 50 \\
Number of CA & 1 \\
Number of SMs & 5 \\
Number of groups & 5 \\
Communication range of loT & $100 \mathrm{~m}$ \\
device & \\
Mobility model & Random Way Point \\
Mobility speed & $10 \mathrm{~m} / \mathrm{s}$
\end{tabular}




\begin{tabular}{ll} 
Total number of packets & 100 \\
Packet size & 1000 bytes (Max) \\
Packet interval & $1 \mathrm{~s}$ \\
Initial energy level & 100 Joules \\
Number of retransmissions & 7 \\
Data rate & $1 \mathrm{Mbps}$ \\
GA-FFA Initial population & 100 \\
& 0.7 \\
$\quad$ Crossover rate & 0.001 \\
$\quad$ Mutation rate & $1.0,0.2$ \\
$\quad \gamma, \beta_{0}$ & 50 \\
Number of iteration & $35 \mathrm{~s}$ \\
\hline
\end{tabular}

In table.1, all significant simulation parameters considered in secure MANET-IoT implementation is illustrated. These parameters are not limited and all other parameters that support MANET-IoT are also considered in our work.

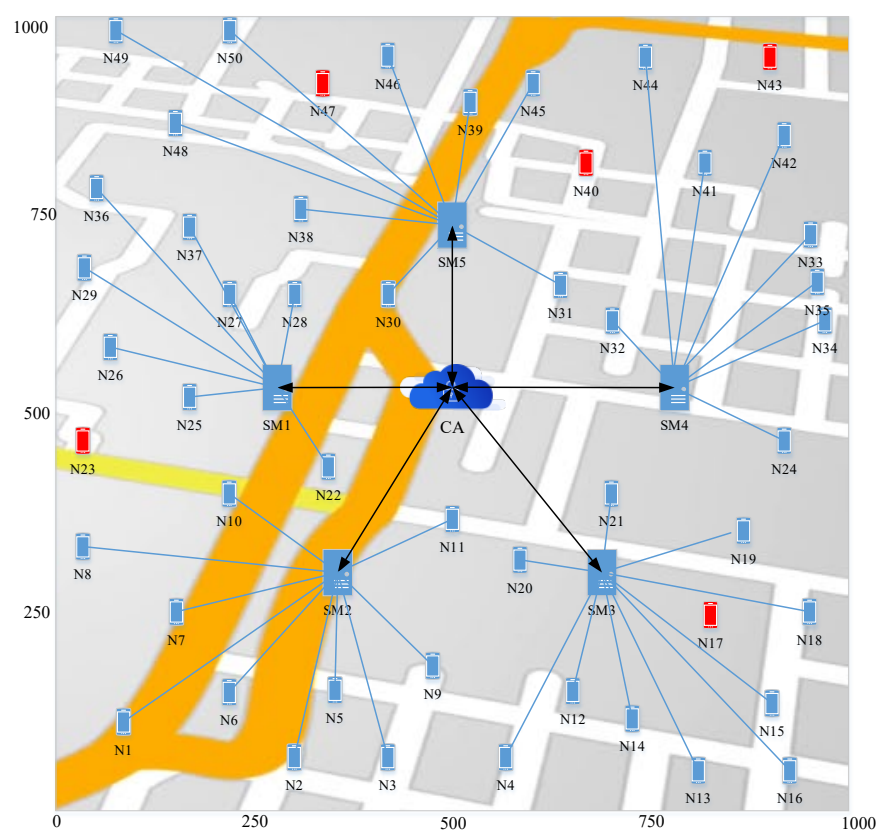

Figure 5. Simulation environment of MANET-IoT

In figure.5, the simulation environment of our proposed MANET-IoT is illustrated for smart city applications. Here the devices include mobile devices of humans, smart devices deployed on animals, vehicles and so on to perform specific operations. Our network environment comprises MANET-IoT devices which move around within the network area and malicious nodes which are looking for launching attack on communication between honest nodes. Here 5 nodes are malicious and those nodes are differentiated from honest nodes using red color. Based on SMs communication range, five groups are formed without malicious nodes.

\subsection{Obtained results in security provision}

Our proposed work achieves data security and integrity by a novel HMAC-AES algorithm. In this subsection, we provide security analysis of proposed work.

Table 2. Security analysis

\begin{tabular}{ll}
\hline Parameter & Value \\
\hline Key size & $128 \mathrm{bit}$ \\
Number of rounds in AES & 10 \\
Block size & 4 \\
Average encryption time & $4 \mathrm{~ms}$ \\
Average decryption time & $5 \mathrm{~ms}$ \\
Number of malicious nodes & $5(10 \%$ of total nodes $)$ \\
Attacks considered & Blackhole and Grayhole \\
\hline
\end{tabular}

In table.2, the security results obtained by our proposed work are depicted. In the network, each node is registered with $\mathrm{CA}$ and obtains unique certificate from $\mathrm{CA}$. The FL_TV computed by RF-K means algorithm is depicted in figure.6. Here we can see that the malicious nodes such as $N 17, N 23, N 40, N 43, N 47$ are given with lower trust values than other honest nodes. Involvement of flawless trust computation improves the trust value computation in our work.

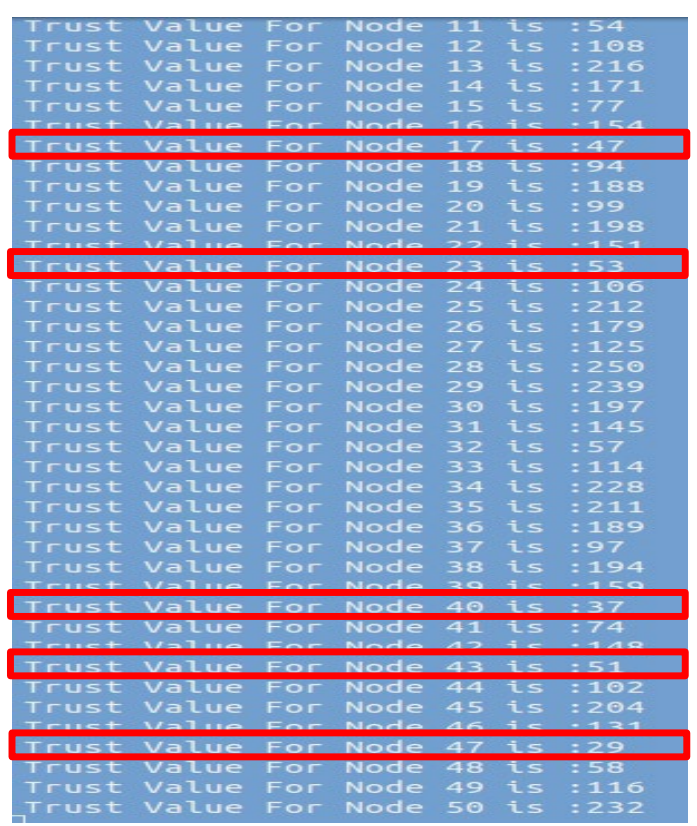

Figure 6. Simulation environment of MANET-IoT

Then our proposed security algorithms such as SCGF algorithm, RF-K means algorithm, GA-FFA algorithm and HMAC-AES algorithm are performed in MANETIoT in order to improve network security for various IoT applications. 


\subsection{Performance Evaluation and Comparative analysis}

In this sub-section, we evaluate our proposed work in MANET-IoT network based on following performance metrics: packet delivery ratio (PDR), throughput, delay, and malicious activity detection. Our proposed work is compared with state-of-the-art works such as SOADV [28], FBSA [27], and conventional AODV. The performance metrics considered in our work are described as follows,

- PDR: PDR is defined as the ratio between number of packets transmitted by source node and the number of packets received by destination node successfully.

- Throughput: throughput is defined as the average rate of successful data transmission over a communication link in the network at given period of time.

- Delay: In the network, delay is defined as the average time taken by a data packet to reach the destination from source node.

- Malicious activity detection: This metric is defined as the ratio between number of malicious nodes identified by security scheme to the total number of malicious nodes in the network.

- Energy Consumption: In the network, energy consumption is estimated by computing energy consumed due to data transmission, data reception, and idle listening.

Table 3. Comparative Analysis

\begin{tabular}{|c|c|c|c|c|}
\hline Parameter & AODV & SAODV & FBSA & Proposed \\
\hline $\begin{array}{l}\text { Routing } \\
\text { algorithm }\end{array}$ & AODV & S-AODV & OLSR & GA-FFA \\
\hline $\begin{array}{l}\text { Route } \\
\text { selection } \\
\text { metrics }\end{array}$ & Distance & Distance & $\begin{array}{l}\text { PDR \& } \\
\text { Delay }\end{array}$ & $\begin{array}{l}\text { Flawless } \\
\text { trust, } \\
\text { residual } \\
\text { energy, } \\
\text { link } \\
\text { stability, } \\
\text { hop } \\
\text { count }\end{array}$ \\
\hline $\begin{array}{l}\text { Security } \\
\text { metrics }\end{array}$ & None & None & Trust & $\begin{array}{l}\text { Flawless } \\
\text { Trust }\end{array}$ \\
\hline $\begin{array}{l}\text { Data } \\
\text { security }\end{array}$ & $\begin{array}{l}\text { Not } \\
\text { provided }\end{array}$ & $\begin{array}{l}\text { Not } \\
\text { provided }\end{array}$ & $\begin{array}{l}\text { Not } \\
\text { provided }\end{array}$ & $\begin{array}{l}\text { Data is } \\
\text { secured }\end{array}$ \\
\hline $\begin{array}{l}\text { Security } \\
\text { algorithm }\end{array}$ & None & None & None & $\begin{array}{l}\text { HMAC- } \\
\text { AES }\end{array}$ \\
\hline $\begin{array}{l}\text { Trust } \\
\text { mechanism }\end{array}$ & None & None & $\begin{array}{l}\text { Based } \\
\text { on PDR } \\
\text { and } \\
\text { delay }\end{array}$ & $\begin{array}{l}\text { RF-K } \\
\text { means }\end{array}$ \\
\hline
\end{tabular}

In table.3, comparative analysis between proposed work and state-of-the-art works has depicted. The analysis confirms that proposed work fulfills all security and routing requirements better than previous works.

\section{Analysis on PDR}

PDR is a significant metric that measures successful data transmissions held over the network. In the presence of malicious nodes, the packet loss is increased in the network which minimizes the PDDR.

In figure.7, we compare PDR obtained by proposed work with previous research works. Here we can see that, our proposed work achieves better PDR than previous works. When average speed of nodes is $2 \mathrm{~m} / \mathrm{s}$, $96 \%$ of packets are transmitted to destination successfully in our work. In the same node speed, FBSA method transmits $94 \%$ of packets to destination and SAODV method transmits $36 \%$ of packets successfully to destination. Here SADOV method and AODV protocol are fail to provide better even upto $50 \%$. In addition, FBSA method also provides PDR 2\% lower than proposed work. This analysis shows that involvement of effectual security schemes and optimal routing in proposed work improves PDR of the network. The previous works are not able to achieve better PDR since the involvement of adversaries which limits the network performance.

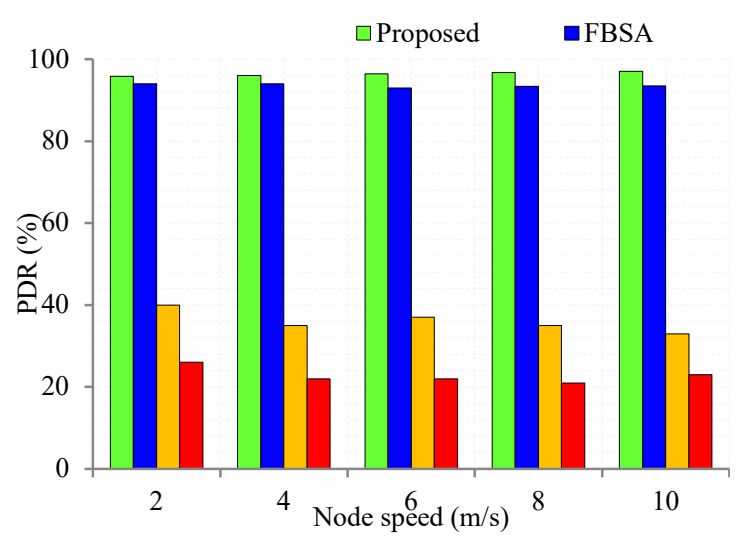

Figure 7. Comparative analysis on PDR

\section{Analysis on throughput}

Network throughput is an significant metric that measures the performance of entire network. In the presence of adversaries this metric is low (i.e.) in a secure environment throughput is large.

In figure.8, throughput metric achieved by our proposed work is compared with previous works. Our proposed work achieves throughput upto $140 \mathrm{kbps}$ even in high mobility (i.e.) $10 \mathrm{~m} / \mathrm{s}$. perhaps, the modes are highly dynamic; selection optimal route by GA-FFA algorithm and group formation by SCGF algorithm improves the performance of the network. In addition, formulation of $F L_{-} T V$ helps in detection of highly secure route which further improve network performance. In FBSA method, the throughput is only $121 \mathrm{kbps}$ which is $20 \mathrm{kbps}$ lower than our proposed approach. Absence of secure routing 
and mobility management scheme results in lower throughput performance. But conventional AODV and AODV protocols are not able to handle large number of adversaries and high dynamic mobility. Thus both protocols are not able to improve network throughput.

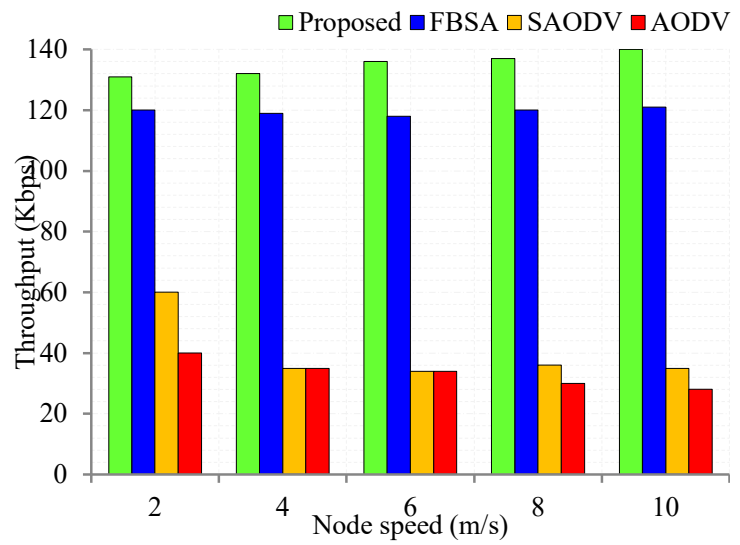

Figure 8. Comparative analysis on throughput

\section{Analysis on delay}

In mobile network environment, delay is significant since it is increased with increase in node mobility. Thus this analysis will show the ability of the work to cope with dynamic environment.

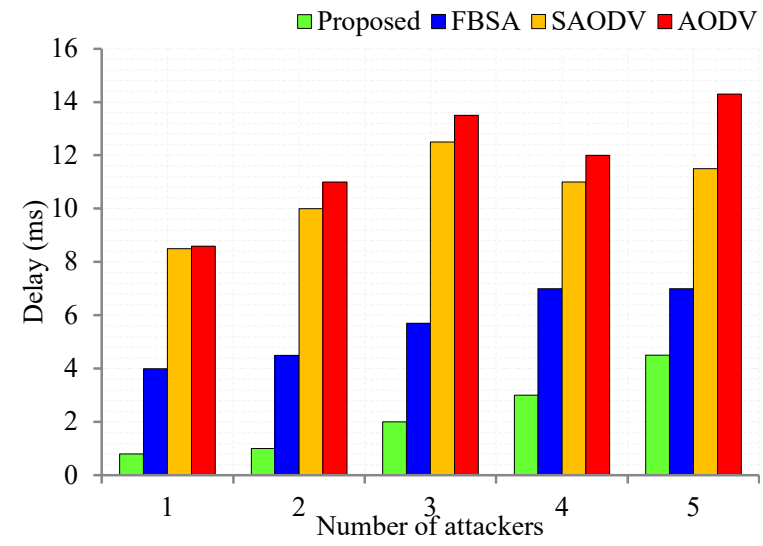

Figure 9. Comparative analysis on delay

In figure.9, proposed work is analyzed in terms of delay metric. The analysis shows that proposed work minimizes delay (i.e.) in our proposed work the data is transmitted to destination from source node within minimum transmission time. The delay is increased with increase in number of attackers. This is because; involvement of attackers disrupts data transmission through packet dropping, data redirection and so on. However, our proposed work improves delay even in the presence of five malicious nodes. The reason behind improved delay in our work is that involvement of FL_TV, secure grouping, and secure routing minimizes delay metric in the network. The previous methods results in large time delay which degrades the performance of the network. This analysis confirms that FBSA, SAODV methods are not able secure network environment. the results obtained for AODV protocol shows that absence of security scheme results in increased adversaries which not suitable for mobile environment. in the presence of five attackers, proposed work provides $4.5 \mathrm{~ms}$ of delay which is relative lower than previous works.

\section{Table 4. Efficiency of proposed work in data} transmission

\begin{tabular}{llll}
\hline Method & PDR (\%) & Throughput (kbps) & $\begin{array}{l}\text { Delay } \\
(\mathrm{ms})\end{array}$ \\
\hline AODV & 23 & 32 & 14 \\
SAODV & 36 & 40 & 13 \\
FBSA & 93 & 119 & 7.6 \\
Proposed & 96.3 & 135 & 3.26 \\
\hline
\end{tabular}

In table.4, efficiency of our proposed work in data transmission is analyzed in terms of performance metrics. From the analysis we can conclude that proposed work achieves better performance in data transmission. Involvement of secure group formation supports mobility management while involvement of FL_TV formulation relaxes the problem of adversaries in data transmission. In addition, secure routing and secure data transmission protect the data from adversaries which increases number of retransmissions. Thus each significant process involved in our work contributes in efficient data transmission in MANET-IoT environment.

\section{Analysis on malicious activity detection}

The prime objective of our work is to minimize the presence of adversaries in the network. To analyze the security strength of our proposed work, we analyze malicious activity detection rate.

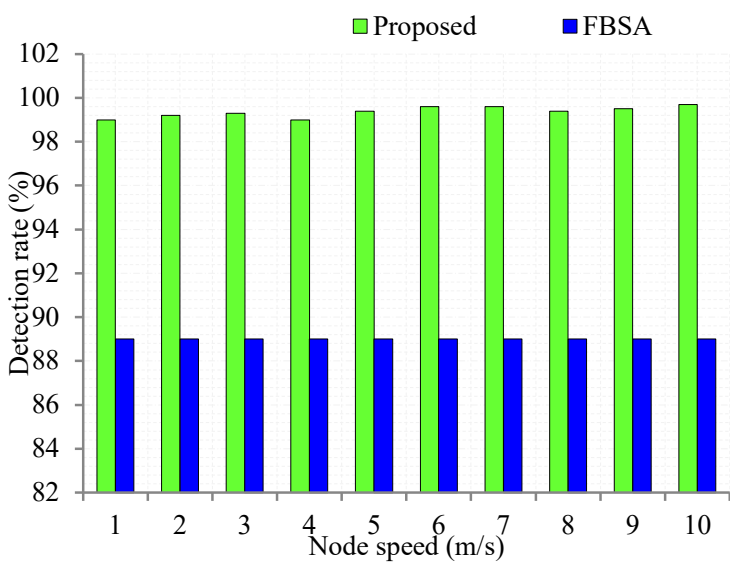

Figure 10. Comparative analysis on malicious activity detection 
In figure.10, detection rate achieved by proposed work and previous FBSA method is compared. The graphical illustration shows that our proposed work is effectual in malicious activity detection. Averagely, our proposed work achieves $10 \%$ better detection rate than FBSA method. In our work, $99 \%$ of malicious nodes are identified accurately whereas in FBSA method only $89 \%$ of malicious nodes are detected correctly. Both methods utilize trust value for malicious activity detection. However, our proposed work improves detection rate with significant trust value computation by flawless trust formulation. In addition, secure route selection also minimizes involvement of adversaries in the network. Thus with the support of FL_TV formulation, our proposed work improves malicious activity detection rate upto $96 \%$.

Table 5. Analysis on malicious node detection

\begin{tabular}{lll}
\hline Parameter & FBSA & Proposed \\
\hline Number of nodes & 50 & 50 \\
Number of malicious node & 5 & 5 \\
$\begin{array}{l}\text { Number of malicious nodes } \\
\text { identified accurately }\end{array}$ & $\cong 4$ & $\cong 5$ \\
Detection rate & $89 \%$ & $99 \%$ \\
\hline
\end{tabular}

In table.5, the detection rate achieved by proposed work and FBSA method is analyzed. In our work, $99 \%$ of malicious nodes are detected accurately (i.e.) all 5 malicious nodes are identified accurately. But in FBSA method, 4 nodes only detected as malicious nodes. Thus our proposed work is efficient in attack detection rate.

\section{Analysis on energy consumption}

In literature, security provisioning approaches often increases energy consumption. In this subsection, energy consumption is analyzed with respect to malicious activities.

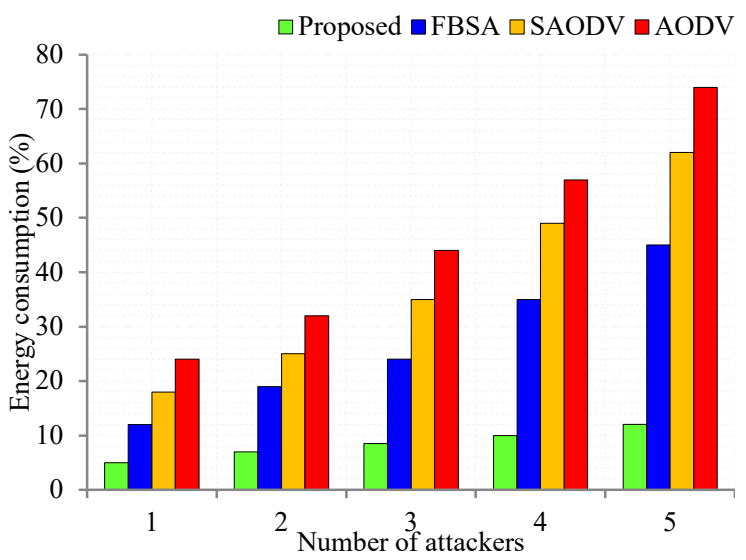

Figure 11. Comparative analysis on energy consumption
In figure.11, energy consumption is analyzed based on number of attacker nodes in the network. In the graph, we can see that proposed work consumes only $12 \%$ of energy even with 5 attackers. However, for the same number of attackers FBSA scheme consumes nearly $45 \%$ of energy due to absence of effectual security scheme. Likewise, SAODV and AODV consume $62 \%$ and $74 \%$ of energy respectively. The major reason for this huge energy difference is, previous approaches require large number of retransmissions due to involvement of malicious nodes. But in proposed work, malicious nodes are identified accurately and optimum path is selected for route election. Thus combined security provision and optimal route selection minimizes energy consumption in the network.

Table 6. Analysis on energy consumption

\begin{tabular}{lll}
\hline Method & Initial energy $(\mathrm{J})$ & $\begin{array}{l}\text { Average Energy } \\
\text { consumption (\%) }\end{array}$ \\
\hline AODV & 100 & 46.2 \\
SAODV & 100 & 37.8 \\
FBSA & 100 & 27 \\
Proposed & 100 & 8.5 \\
\hline
\end{tabular}

In table.6, average energy consumption metric is compared with for each work. The analysis shows that proposed work achieves relatively minimum energy consumption $(8.5 \%)$ which is $17 \%$ lower than FBSA method, 29\% lower than SAODV, and 37\% lower than conventional AODV scheme.

\section{Conclusion}

In this paper, a novel security scheme is proposed with flawless trust formulation mechanism for MANET-IoT environment. To improve network management, the network is initially grouped by SCGF algorithm in secure manner. In each secure group, flawless trust computation is performed by SM using RF-K means algorithm. Flawless trust computation uses recommendation filtering approach which also helps in Grayhole attack detection. For secure as well as effectual data transmission, GA-FFA based optimal route selection algorithm is presented. Here GA-FFA algorithm utilizes a new fitness value that is formulated by flawless trust value and significant routing metrics. The transmitted data is secured by HMAC-AES algorithm that combines cryptography function and hash function together. HMAC-AES algorithm also assures data integrity which is significant for mobile environment. Experimental results show that proposed work is effectual in PDR, throughput, delay, malicious activity detection, and energy efficiency. From the achieved results, it is clear that proposed work better in network management as well as security. On the whole, our proposed work achieves data security and integrity with improved data transmission in MANET-IoT environment. In future, we 
have planned to extend this work with energy efficient schemes. Although the results have shown that proposed work achieves better energy efficiency we aim to improve further energy efficiency of the network without loss in security level.

\section{References}

[1] Leite, J.R., Ursini, E.L., \& Martins, P.S. (2017). Simulation of AdHoc Networks Including Clustering and Mobility. ADHOC-NOW.

[2] Anjum, S.S., Noor, R.M., \& Anisi, M.H. (2017). Review on MANET Based Communication for Search and Rescue Operations. Wireless Personal Communications, 94, 31-52.

[3] Kandari, S., \& Pandey, M.K. (2016). Impact of Residual Life Estimator Battery Model on QoS Issues in MANET. Wireless Personal Communications, 86, 601-614.

[4] Giri, A., Dutta, S., Neogy, S., Dahal, K., \& Pervez, Z. (2017). Internet of Things (IoT): A Survey on Architecture, Enabling Technologies, Applications and Challenges. International Conference on Internet of Things and Machine Learning.

[5] Razzaque, M.A., Milojevic-Jevric, M., Palade, A., \& Clarke, S. (2016). Middleware for Internet of Things: A Survey. IEEE Internet of Things Journal, 3, 70-95.

[6] Jabbar, W.A., Saad, W.K., \& Ismail, M. (2018). MEQSAOLSRv2: A Multicriteria-Based Hybrid Multipath Protocol for Energy-Efficient and QoS-Aware Data Routing in MANET-WSN Convergence Scenarios of IoT. IEEE Access, 6, 76546-76572.

[7] Bujari, A., Furini, M., Mandreoli, F., Martoglia, R., Montangero, M., \& Ronzani, D. (2018). Standards, Security and Business Models: Key Challenges for the IoT Scenario. Mobile Networks and Applications, 23, 147-154.

[8] Liu, G., Yan, Z., \& Pedrycz, W. (2018). Data collection for attack detection and security measurement in Mobile Ad Hoc Networks: A survey. J. Network and Computer Applications, 105, 105-122.

[9] Gurung, S., \& Chauhan, S. (2018). A novel approach for mitigating gray hole attack in MANET. Wireless Networks, 24, 565-579.

[10] Gupta, P., Goel, P., Varshney, P., \& Tyagi, N. (2019). Reliability Factor Based AODV Protocol: Prevention of Black Hole Attack in MANET.

[11] Dorri, A. (2017). An EDRI-based approach for detecting and eliminating cooperative black hole nodes in MANET. Wireless Networks, 23, 1767-1778.

[12] Airehrour, D., Gutiérrez, J.A., \& Ray, S.K. (2016). Securing RPL routing protocol from blackhole attacks using a trust-based mechanism. 2016 26th International Telecommunication Networks and Applications Conference (ITNAC), 115-120.

[13] Ahmed, M.N., Abdullah, A.H., Chizari, H., \& Kaiwartya, O. (2017). F3TM: flooding factor based trust management framework for secure data transmission in MANETs.

[14] Kushwaha, A., Sharma, H.R., \& Ambhaikar, A. (2016). A Novel Selective Encryption Method for Securing Text Over Mobile Ad Hoc Network.

[15] Jain, A., Tokekar, V., \& Shrivastava, S.K. (2018). Security Enhancement in MANETs Using Fuzzy-Based Trust Computation Against Black Hole Attacks.

[16] Panos, C., Ntantogian, C., Malliaros, S., \& Xenakis, C. (2017). Analyzing, quantifying, and detecting the blackhole attack in infrastructure-less networks. Computer Networks, 113, 94-110.

[17] Singh, O., Singh, J., \& Singh, R. (2017). Multi-level trust based intelligence intrusion detection system to detect the malicious nodes using elliptic curve cryptography in MANET. Cluster Computing, 1-13.

[18] Schweitzer, N., Stulman, A., Margalit, R.D., \& Shabtai, A. (2017). Contradiction Based Gray-Hole Attack Minimization for Ad-Hoc Networks. IEEE Transactions on Mobile Computing, 16, 2174-2183.

[19] Garg, M.K., Singh, N., \& Verma, P. (2018). Fuzzy rulebased approach for design and analysis of a Trust-based Secure Routing Protocol for MANETs. Elsevier, 132, 653658.

[20] Gomathi, K., Parvathavarthini, B., \& Saravanakumar, C. (2017). An Efficient Secure Group Communication in MANET Using Fuzzy Trust Based Clustering and Hierarchical Distributed Group Key Management. Wireless Personal Communications, 94, 2149-2162.

[21] Shams, E.A., \& Rizaner, A. (2018). A novel support vector machine based intrusion detection system for mobile ad hoc networks. Wireless Networks, 24, 1821-1829.

[22] Song, T., Li, R., Mei, B., Yu, J., Xing, X., \& Cheng, X. (2016). A Privacy Preserving Communication Protocol for IoT Applications in Smart Homes. IEEE Internet of Things Journal, 4, 1844-1852.

[23] Li, X., Niu, J., Bhuiyan, M.Z., Wu, F., Karuppiah, M., \& Kumari, S. (2018). A Robust ECC-Based Provable Secure Authentication Protocol With Privacy Preserving for Industrial Internet of Things. IEEE Transactions on Industrial Informatics, 14, 3599-3609.

[24] Li, F., Zheng, Z., \& Jin, C. (2016). Secure and efficient data transmission in the Internet of Things. Telecommunication Systems, 62, 111-122.

[25] Kumar, V.V., \& Ramamoorthy, S.K. (2018). Secure Adhoc On-Demand Multipath Distance Vector Routing in MANET. International Conference on Computing and Communications Systems, Springer, pp. 49-63.

[26] Tan, S., Li, X., \& Dong, Q. (2016). A Trust Management System for Securing Data Plane of Ad-Hoc Networks. IEEE Transactions on Vehicular Technology, 65, 75797592.

[27] Bisen, D., \& Sharma, S. (2018). Fuzzy Based Detection of Malicious Activity for Security Assessment of MANET. National Academy Science Letters, 41, 23-28.

[28] Jain, A.K., \& Tokekar, V. (2015). Mitigating the effects of Black hole attacks on AODV routing protocol in mobile ad hoc networks. 2015 International Conference on Pervasive Computing (ICPC), 1-6.

[29] Yen, Y., Chan, Y., Chao, H., \& Park, J.H. (2008). A genetic algorithm for energy-efficient based multicast routing on MANETs.

[30] Persis, D.J., \& Robert, T.P. (2016). Reliable Mobile AdHoc Network Routing Using Firefly Algorithm.

[31] Sumra, I.A., Sellappan, P., Abdullah, A.V., \& Ali, A. (2018). Security issues and Challenges in MANETVANET-FANET: A Survey. EAI Endorsed Transactions on Energy Web and Information Technologies, 5 (17).

[32] Wang, P., \& Zhang, P. (2016). A Review on Trust Evaluation for Internet of Things. MobiMedia.

[33] Kumar, A., Vishnoi, P., \& ShimiS., L. (2019). Smart Grid Security with Cryptographic Chip Integration. EAI Endorsed Trans. Energy Web, 6, e6.

[34] Li, X., Lyu, M.R., \& Liu, J. (2004). A trust model based routing protocol for secure ad hoc networks. 2004 IEEE 
Aerospace Conference Proceedings (IEEE Cat. No.04TH8720), 2, 1286-1295 Vol.2.

[35] Liu, K., Deng, J., Varshney, P.K., \& Balakrishnan, K. (2007). An Acknowledgment-Based Approach for the Detection of Routing Misbehavior in MANETs. IEEE Transactions on Mobile Computing, 6 . 